\title{
Adaptação de Jogos Sérios para Crianças com Deficiência Física nos Membros Superiores
}

\author{
Flávia Gonçalves Fernandes', Alexandre Cardoso', \\ Edgard Afonso Lamounier Júnior ${ }^{1}$ \\ ${ }^{1}$ Faculdade de Engenharia Elétrica - Universidade Federal de Uberlândia (UFU) \\ Uberlândia, MG - Brasil \\ flavia.fernandes92@gmail.com, alexandre@ufu.br, lamounier@ufu.br
}

\begin{abstract}
The development of information technology has been helping numerous practices in education and health, in activities such as diagnosis, treatment, management and learning, which requires the need for change and development of new skills by professionals of the areas involved. In this perspective, the objective of this work is to adapt serious educational games for children with physical disabilities in the upper limbs. As methodology, the resources of the Virtual Reality were used in addition to the features of Myo wearable device to control serious games. Soon, it is expected to contribute to the accessibility and motivation of these children, through technological innovations, creating a learning environment and more interactive, playful and attractive rehabilitation, also making it easier acceptance of disability.
\end{abstract}

Resumo. O desenvolvimento de tecnologias da informação vem auxiliando inúmeras práticas na área da educação e da saúde, em atividades como diagnóstico, terapia, gerenciamento e aprendizagem, o que exige a necessidade de mudanças e desenvolvimento de novas habilidades pelos profissionais das áreas envolvidas. Nessa perspectiva, o objetivo deste trabalho é adaptar serious games educativos para crianças com deficiência fisica nos membros superiores. Como metodologia, foram utilizados os recursos da Realidade Virtual, além das funcionalidades do dispositivo vestível Myo para controlar os serious games. Logo, espera-se contribuir na acessibilidade e motivação dessas crianças, através das inovações tecnológicas, gerando um ambiente de aprendizagem e reabilitação mais interativo, lúdico e atrativo, também tornando mais fácil a aceitação da deficiência.

\section{Introdução}

A reabilitação é um procedimento que exige o conhecimento sobre a enfermidade a ser tratada e as deficiências causadas no movimento. A cada dia, torna-se mais evidente a necessidade de embasamento científico sobre o corpo humano e suas respostas fisiológicas para uma consistente tomada de decisão, visando a recuperação das funções motoras, prevenir e reduzir os riscos de novas lesões. A intensidade e o volume de tratamento e treinamento são fundamentais para a obtenção de resultados positivos. A quantidade de sessões semanais, duração das sessões e duração total do tratamento são fundamentais para a otimização das respostas [Monteiro Junior et al., 2011].

O longo tempo necessário para o tratamento e a pouca motivação gerada pelos métodos tradicionais são apontados como motivo de abandono do tratamento 
fisioterápico, caracterizando-se como uma das principais causas de falha terapêutica. A reabilitação convencional é, por natureza, repetitiva e muitas vezes dolorosa. Outra característica é a falta de recursos que possibilitem dar continuidade do tratamento domiciliar, com pouco ou nenhum monitoramento presencial de um terapeuta [Dias et al., 2009].

Assim, jogos de reabilitação abrem novas possibilidades, onde exercícios físicos de reabilitação podem ser realizados em casa pelo paciente, com ou sem a presença do fisioterapeuta. A ideia da reabilitação virtual é que o paciente siga as instruções de exercícios do jogo corretamente, realizando os exercícios em casa e retorne ao centro de reabilitação uma ou duas vezes por semana para monitorar a evolução do tratamento e receber novas orientações sobre exercícios, quando necessário [Grande et al., 2011].

Dessa forma, os sistemas de realidade virtual têm se mostrado bastante eficientes no campo da medicina, pois além de tornar o tratamento mais divertido e motivador, torna a execução dos exercícios feitos em domicílio mais segura, pois terá maior precisão de movimentos com ou sem a supervisão de um terapeuta. A forma e velocidade correta com que devem ser executados os exercícios, são orientados durante as sessões de fisioterapia, onde o fisioterapeuta pode avaliar se o paciente está apto para utilizar um tratamento de reabilitação virtual em domicílio, para proporcionar mais tranquilidade ao terapeuta e comodidade ao paciente, pois torna os resultados mais eficazes e precisos [Santarosa et al., 2012].

Nessa linha de raciocínio, o objetivo deste trabalho é adaptar serious games, que utilizam Realidade Virtual e dispositivo vestível Myo, para crianças e adolescentes com faixa etária de seis a quinze anos com deficiência física nos membros superiores, a fim de que eles tenham maior acessibilidade a jogos educativos e melhor aceitação da deficiência.

Enfim, espera-se que os resultados deste estudo possam contribuir, de alguma forma, com as discussões atuais e futuras em torno da temática sobre o uso de serious games utilizando Realidade Virtual e dispositivos vestíveis (wearables) para auxiliar no processo de reabilitação de pessoas deficientes.

\section{Trabalhos Relacionados}

Dentre os trabalhos disponíveis na literatura, alguns deles apresentam aplicações utilizando o dispositivo vestível Myo.

O trabalho "Myo Armband for Physiotherapy Healthcare: A Case Study Using Gesture Recognition Application" apresenta o o "Diagnostics Myo", um aplicativo baseado em computador para compreender gráficos de eletromiografia (EMG), bits de dados vetoriais e sinais elétricos do braço humano. Essa análise é útil para detectar anormalidades médicas e movimentos da mão, auxiliando em tratamentos fisioterapêuticos e diversas doenças [Sathiyanarayanan e Rajan, 2016].

Na Figura 1, é apresentada a tela do Diangostics Myo. 
V Congresso Brasileiro de Informática na Educação (CBIE 2016)

Anais dos Workshops do V Congresso Brasileiro de Informática na Educação (CBIE 2016)

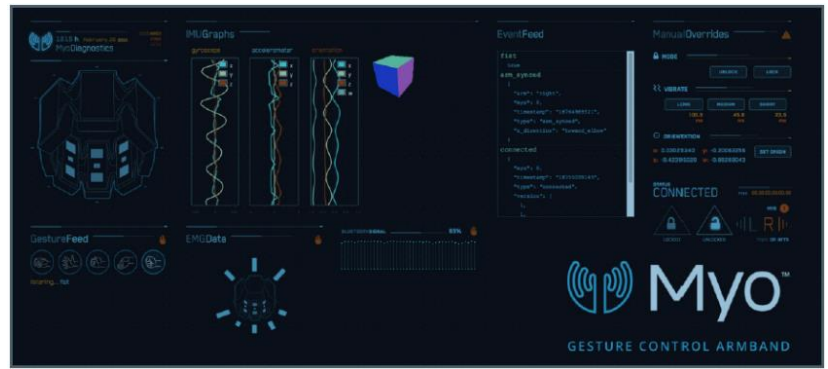

Figura 1: Diagnostics Myo.

O protótipo "MuMyo - Evaluating and Exploring the Myo Armband for Musical Interaction" avalia o potencial do Myo para o desenvolvimento de novas tecnologias para expressão musical para ser usado num contexto NIME (New Interfaces for Musical Expression) [Nymoen et al., 2015].

Na Figura 2, são exibidos testes do protótipo MuMyo com usuários.

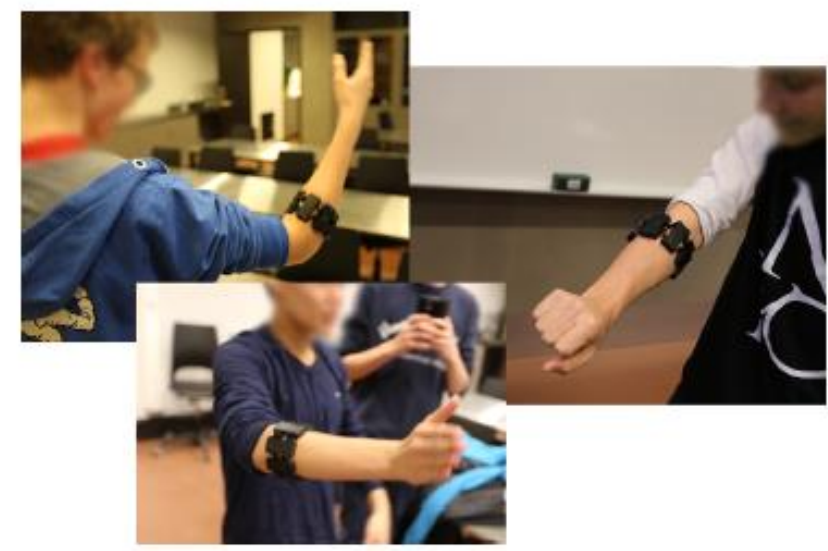

Figura 2: Testes do protótipo MuMyo com usuários.

A pesquisa "Hand Therapist: a rehabilitation approach based on wearable technology and video gaming" trata-se de um sistema de reabilitação da mão, principalmente para pacientes vítimas de acidente vascular cerebral (AVC), composto por: braçadeira Myo, luva robótica e a game engine Unity 3D. Esta abordagem apresenta uma solução que combina desempenho, baixo custo e motivação para terapia da mão [Lipovský e Ferreira, 2015].

A Figura 3 mostra o sistema de terapia de mão em funcionamento.

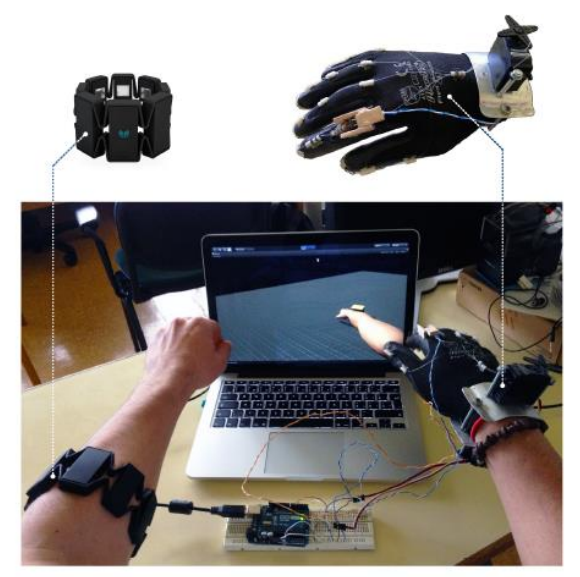

Figura 3: Sistema de terapia de mão em funcionamento. 
V Congresso Brasileiro de Informática na Educação (CBIE 2016)

Anais dos Workshops do V Congresso Brasileiro de Informática na Educação (CBIE 2016)

\section{Metodologia e Tecnologias Utilizadas}

Neste trabalho, foi investigado se a tecnologia de Realidade Virtual juntamente com dispositivos vestíveis (wearables), aplicados ao contexto de serious games para pessoas com deficiência física, têm potencial para auxiliá-las na reabilitação e avaliar o comportamento das pessoas ao utilizarem o sistema.

As atividades e ferramentas utilizadas para o desenvolvimento deste trabalho foram: a pesquisa sobre os tipos de deficiência física nos membros superiores; o estudo e seleção de jogos para adaptá-los para pessoas deficientes; interação de jogos através do dispositivo vestível Myo; testes com os pacientes para coleta de dados; e análise sobre os resultados obtidos.

Dispositivos portáteis ou vestíveis, também conhecidos como wearables, são pequenos dispositivos eletrônicos que podem ser facilmente acoplados ao corpo do usuário. É uma tecnologia de interface avançada entre um usuário e um sistema computacional [Mann, 2012].

O Myo é utilizado no braço ou antebraço do usuário, conforme pode ser observado na Figura 4. É necessário realizar uma calibração da braçadeira para cada usuário de maneira individual, pois cada um possui atividades e contrações musculares específicas.

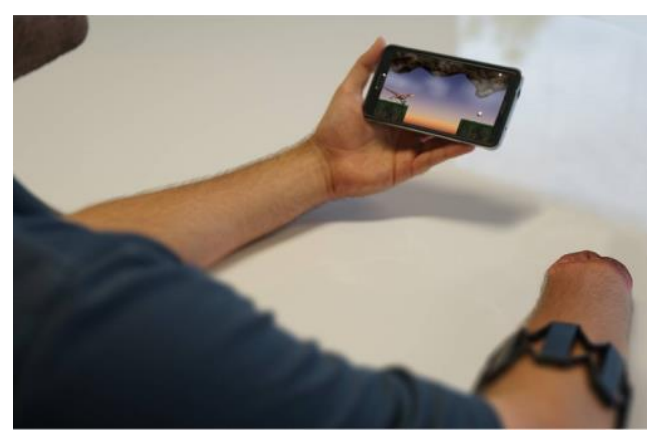

Figura 4: Wearable Myo.

Após efetuada a sua calibração, o wearable possibilita controlar softwares e outras aplicações por meio de gestos e movimentos. Seu propósito é controlar computadores, telefones e outros dispositivos, enviando os dados capturados por ele via Bluetooth. Além disso, não exige câmeras para rastrear os movimentos da mão ou braço e possuem baixo custo [Nuwer, 2013].

Myo é um bracelete de reconhecimento de gestos, que permite o controle de aplicativos e dispositivos sem a necessidade de interagir com nenhum outro periférico. $\mathrm{O}$ Myo utiliza os mesmos parâmetros de sinais mioelétricos que são utilizadas em próteses de braços. Além do grande interesse na utilização da ferramenta para diversas aplicações, vários conectores já foram desenvolvidos e podem ser baixados gratuitamente no Myo play store. O mesmo é compatível com os mais populares sistemas operacionais e se comunica por Bluetooth, o que torna tal tecnologia propensa a ser rapidamente aceita e usada mundialmente [Nuwer, 2013]. 
V Congresso Brasileiro de Informática na Educação (CBIE 2016)

Anais dos Workshops do V Congresso Brasileiro de Informática na Educação (CBIE 2016)

\section{Tipos de Deficiência Física nos Membros Superiores}

Existem diversos tipos de deficiência física nos membros superiores. Porém, para este trabalho foram analisados os seguintes diagnósticos, com os quais foram realizados testes, posteriormente [Greve, 2007]:

- Agenesia de Mão: É a ausência completa ou parcial do membro, como pode ser visto pode ser observado na Figura 5.

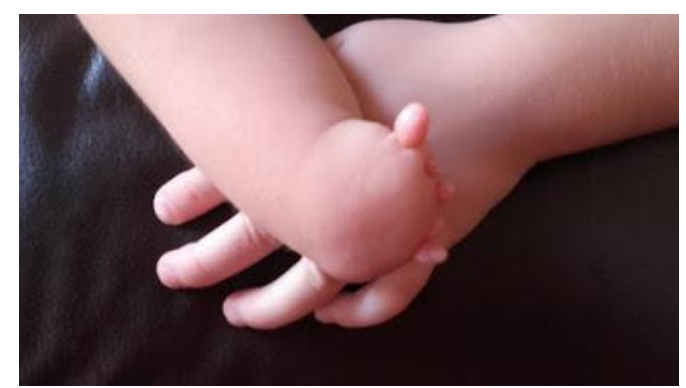

Figura 5: Agenesia de mão.

- Hemimelia de Antebraco: Acontece quando há encurtamento do membro/ausência da extremidade distal do membro ou parte dele isoladamente, de acordo com a Figura 6.

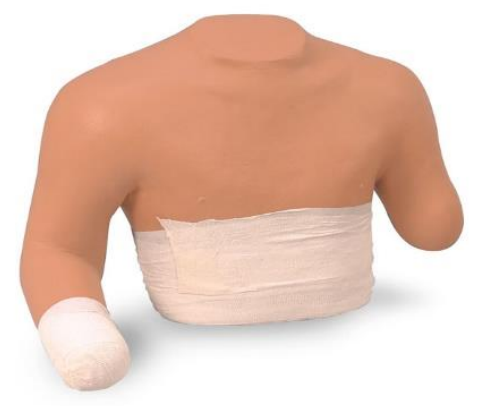

Figura 6: Hemimelia de antebraço.

- Hipoplasia de Falange: Ocorre quando todo o membro (neste caso, os dedos) ou parte dele apresenta tamanho desproporcional (menor), ou quando há ausência deles, conforme pode ser visualizado na Figura 7.

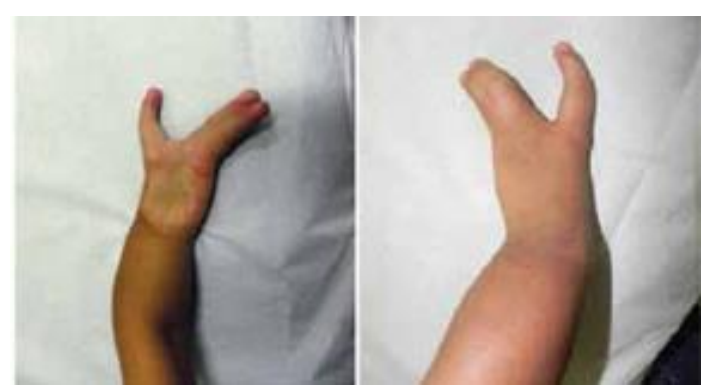

Figura 7: Hipoplasia de falange. 
V Congresso Brasileiro de Informática na Educação (CBIE 2016)

Anais dos Workshops do V Congresso Brasileiro de Informática na Educação (CBIE 2016)

\section{Desenvolvimento}

Gestureworks Gameplay é um aplicativo utilitário leve que permite aos usuários mapear o Myo com poses e gestos para controles de teclado, mouse e diversas outras entradas (inputs). Para isso, cria-se um controlador virtual para um aplicativo ou jogo, que funciona como um perfil de entrada [Myo, 2016].

Na Figura 8, é exibida a tela de configuração das propriedades do aplicativo Gestureworks Gameplay para cadastro dos gestos.

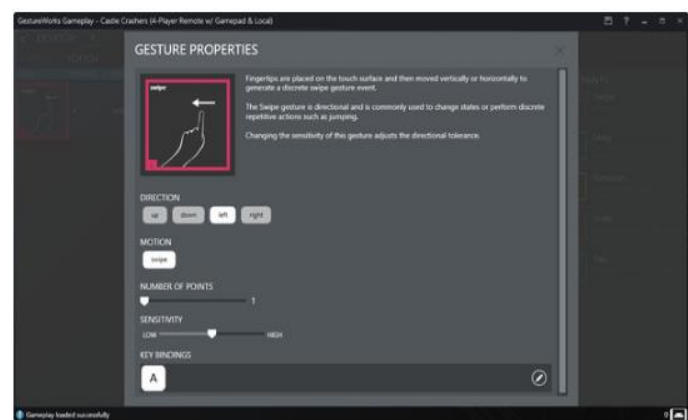

Figura 8: Tela de configuração do Gestureworks Gameplay.

Quando o jogo (ou aplicativo) é executado em segundo plano, juntamente com o Gestureworks Gameplay, permite controlar o jogo utilizando o Myo, ou seja, os jogos que eram comandados por teclado e mouse, são adaptados por meio do aplicativo Gestureworks Gameplay para que se torne possível que pessoas com deficiência física nos membros superiores possam brincar com jogos, superando a dificuldade de interação entre usuário e jogo.

Logo, os controladores virtuais podem ser criados por usuários ou adquiridos por meio de download da biblioteca, permitindo a acessibilidade de pessoas com deficiência física a jogos, melhorando o setor da educação, de lazer e entretenimento para usuários que se enquadram nessa categoria.

O sistema funciona da seguinte maneira: Primeiramente, o usuário coloca o dispositivo vestível Myo em seu braço deficiente para realizar os movimentos necessários durante a execução do jogo e se posiciona em frente a um computador, conforme Figura 9. Ou seja, se o paciente não possui o antebraço direito, ele vai colocar o Myo no seu braço direito, visto que os jogos sérios adaptados neste trabalho têm a finalidade de motivar e treinar o uso de prótese nesses pacientes.

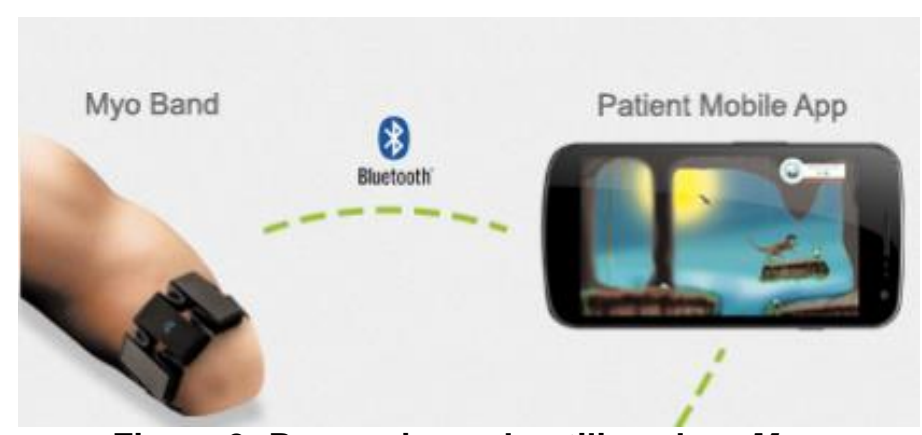

Figura 9: Pessoa jogando utilizando o Myo. 
V Congresso Brasileiro de Informática na Educação (CBIE 2016)

Anais dos Workshops do V Congresso Brasileiro de Informática na Educação (CBIE 2016)

\section{Resultados}

Então, foram adaptados três serious games diferentes, que utilizam o dispositivo Myo como forma de interação, para tornar possível a inclusão de crianças e adolescentes com deficiência física nos membros superiores, os quais são:

- 2048: O jogo consiste de uma tela de $4 \times 4$ quadrados e começa com dois espaços completos com o número 2. O usuário utiliza o dispositivo Myo para controlar o jogo. Desse modo, faz-se movimentos do braço para mover os quadrados de lugar, e os números vão até o fim da linha onde estão. Se o bloco encostar em outro com o mesmo número, eles se somam e viram apenas um bloco de número maior. O objetivo é alcançar um bloco com a soma de 2048. A Figura 10 mostra a tela inicial do jogo 2048.

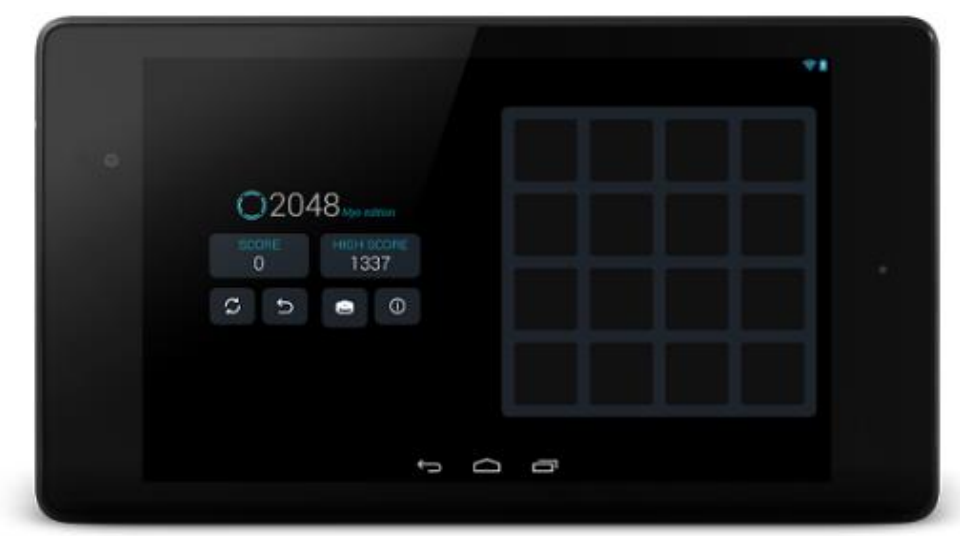

Figura 10: Jogo 2048.

- Minecraft: É um jogo basicamente feito de blocos, tendo as paisagens e a maioria de seus objetos compostos por eles, e permitindo que estes sejam removidos e recolocados em outros lugares para criar construções, empilhandoos. Além da mecânica de mineração e coleta de recursos para construção, há no jogo mistura de sobrevivência, e exploração. Utilizando o Myo, o usuário deve mover o braço para percorrer o cenário e alterar os blocos de lugar, conforme pode ser visto na Figura 11.

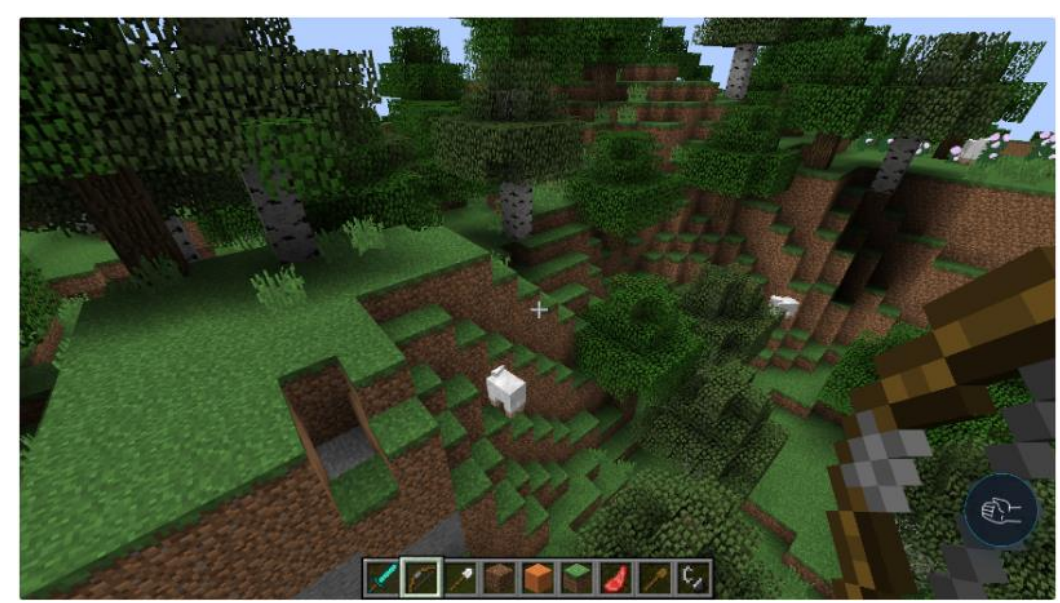

Figura 11: Jogo Minecraft. 
V Congresso Brasileiro de Informática na Educação (CBIE 2016)

Anais dos Workshops do V Congresso Brasileiro de Informática na Educação (CBIE 2016)

- Tetris: É um jogo muito popular, criado em 1984 e jogado por milhões de pessoas em todo o mundo até hoje. Para jogar, o usuário precisa empilhar as pecinhas que vão caindo sem parar. Assim, é preciso eliminar as linhas que se formam com as pecinhas. Utilizando o Myo, o controle desse jogo dá-se por meio de movimentos do braço do usuário, segundo Figura 12.

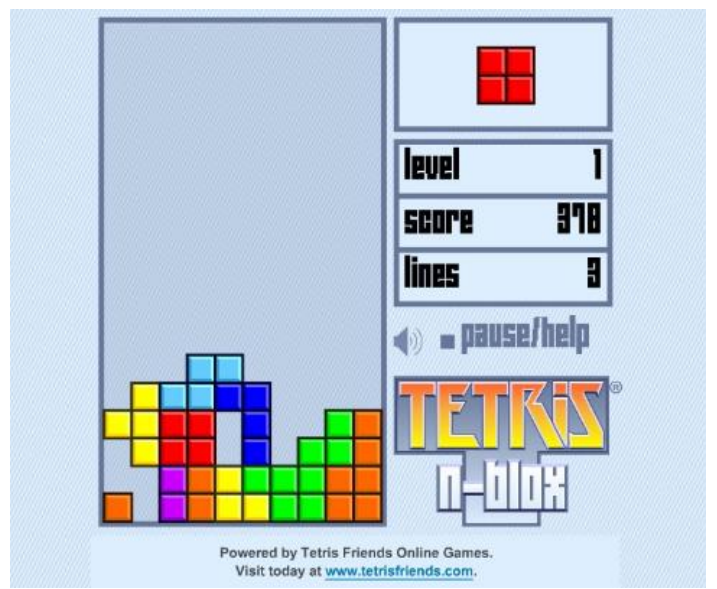

Figura 12: Jogo Tetris.

Após aprovação do projeto pelo Comitê de Ética em Pesquisa (CEP), foram realizados os testes com crianças e adolescentes com deficiência física de uma instituição. Para a realização dos testes, os pais e/ou responsáveis pelas crianças e adolescentes assinaram o Termo de Conhecimento Livre e Esclarecido (TCLE) autorizando a participação de seus filhos para a presente pesquisa.

Na Tabela 1, é apresentada a quantidade de pacientes que participaram dos testes da pesquisa por sexo, faixa etária e deficiência física, totalizando trinta indivíduos, no total, sendo dez pacientes de cada uma das três deficiências físicas abordadas nesse projeto: agenesia de mão, hemimelia de antebraço e hipoplasia de falange.

Tabela 1: Pacientes que participaram da pesquisa.

\begin{tabular}{|c|c|c|c|c|}
\hline Quantidade & \multicolumn{2}{|c|}{ Sexo } & \multicolumn{2}{c|}{ Faixa Etária } \\
\hline Deficiência Física & Masculino & Feminino & $6-10$ anos & $11-15$ anos \\
\hline Agenesia de mão & 6 & 4 & 6 & 4 \\
\hline Hemimelia de antebraço & 5 & 5 & 5 & 5 \\
\hline Hipoplasia de falange & 6 & 4 & 3 & 7 \\
\hline
\end{tabular}

Dentre os jogos selecionados, foram realizados testes utilizando o Myo com crianças e adolescentes que possuem diversos tipos de deficiências físicas nos membros superiores da instituição que realiza o acompanhamento desses pacientes.

O Myo possui a habilidade de controlar aparelhos, sendo possível controlar jogos e vídeos, passar slides e manipular aeromodelos apenas com os movimentos do braço. Esta informação é enviada a um processador na braçadeira, e um algoritmo traduz os comandos, que são enviados via Bluetooth de baixo consumo energético para o gadget que o usuário está tentando controlar, como um smartphone. 
O dispositivo wearable Myo, ao invés de reproduzir diretamente os movimentos, como o Kinect, tem formato de braçadeira e fica posicionado no braço do usuário; através do contato com a pele, percebe a atividade elétrica dos músculos do braço, traduzindo os mínimos movimentos para um computador com a capacidade de controlar uma variedade de aparelhos.

Após a execução dos testes, foram disponibilizados questionários de avaliação para os pacientes responderem. O processo de testes foi acompanhado pela terapeuta ocupacional da instituição, a qual também auxiliou no estágio de escolha dos serious games a serem utilizados e avaliados na pesquisa.

Posteriormente, foi realizada uma análise sobre os resultados encontrados comparando a preferência e a eficácia dos serious games utilizados conforme faixa etária, gênero e tipo da deficiência física.

A partir dos dados obtidos, observou-se que a adaptação dos jogos para crianças e adolescentes com deficiência física nos membros superiores utilizando o dispositivo vestível Myo foi eficaz para possibilitar a inclusão escolar das mesmas, visto que possibilita maior acessibilidade a jogos e a outros programas utilitários do computador.

A técnica utilizada substitui o mouse e o teclado para realização de comandos básicos de entrada de dados, o que facilita a vida das pessoas que possuem dificuldade de manuseio de ferramentas tecnológicas devido à deficiência física nos membros superiores.

Avaliando os questionários respondidos, os usuários do sistema que possuem deficiência física nos membros superiores, gostaram muito dessa inovação tecnológica e se sentiram mais motivados a aprender, uma vez que eles acreditam que essa ferramenta pode ser muito útil para auxiliar o processo de inclusão escolar e social.

\section{Conclusões e Trabalhos Futuros}

Portanto, observa-se que o objetivo principal desse trabalho foi alcançado, uma vez que possibilitou maior inclusão escolar e acessibilidade a jogos educativos, lazer e entretenimento de crianças e adolescentes com deficiência física nos membros superiores por meio do dispositivo vestível Myo. Com isso, os pacientes passaram a se sentir mais incluídos, houve uma maior aceitação da sua limitação física e também melhoraram a sua disposição e motivação em relação ao ensino-aprendizagem e a continuar o tratamento de reabilitação.

As principais dificuldades encontradas na realização deste trabalho foram a modelagem para a construção do jogo e o reconhecimento dos movimentos pelo dispositivo wearable Myo, devido à sua complexidade e à diversidade de variáveis envolvidas neste processo.

Como trabalhos futuros, pretende-se prosseguir com a seleção de mais jogos com a finalidade de melhorar o incentivo ao uso de prótese em crianças com deficiência física nos membros superiores. Além disso, deseja-se pesquisar dispositivos vestíveis para adaptar mais serious games para outros membros do corpo humano. E também realizar o desenvolvimento de um módulo de supervisão da aplicação, no qual o profissional habilitado poderia visualizar gráficos para acompanhar a evolução de determinando paciente, e também comparar os resultados obtidos por diferentes pacientes na execução dos jogos. 
V Congresso Brasileiro de Informática na Educação (CBIE 2016)

Anais dos Workshops do V Congresso Brasileiro de Informática na Educação (CBIE 2016)

\section{Referências}

Dias, R. S.; Sampaio, I. L. A.; Taddeo, L. S. Fisioterapia X WII: “A Introdução do Lúdico no processo de reabilitação de pacientes em tratamento fisioterapêutico". In: VIII Brazilian Symposium on Games and Digital Entertainment. 4. Rio de JaneiroRJ, 2009.

Grande, A. A. B.; Galvão, F. R. O.; Gondim, L. C. A. "Reabilitação virtual através do videogame: relato de caso no tratamento de um paciente com lesão alta dos nervos mediano e ulnar". Revista Acta Fisiátrica, Rio Grande do Norte, v. 18, n. 3, p. 157$162,2011$.

Greve, J. M. D. "Reabilitação: conceito terapêutico". In: Tratado de Medicina de reabilitação. São Paulo: Manole, p 8-9, 2007.

Lipovský, R. e Ferreira H. A. "Hand Therapist: a rehabilitation approach based on wearable technology and video gaming". In: Portuguese BioEngineering Meeting. 4. Porto: Portugal, 2015.

Mann S. "Computação Wearable". In: Soegaard, Mads e Dam, Rikke Friis. Encyclopedia of Interação Humano-Computador. Aarhus, 2012. Dinamarca: A Fundação Interaction-Design.org.

Monteiro Junior et al., R. S. "Efeito da reabilitação virtual em diferentes tipos de tratamento". Revista Brasileira de Ciência da Saúde, Rio de Janeiro, n. 29, p. 56-63, jul/set. 2011.

Myo. "GestureWorks Gameplay". Disponível em: $<$ https://market.myo.com/app/54f47df3e4b076579f2a08fb/gestureworks-gameplay3>. Acesso em: 10 mar. 2016.

Nymoen, K.; Haugen, M. R. e Jensenius, A. R. "MuMyo Evaluating and Exploring the Myo Armband for Musical Interaction". In: Proceedings of the International Conference on New Interfaces for Musical Expression. Los Angeles: EUA, 2015.

Nuwer R. "Armband adds a twitch to gesture control”. New Scientist 217.2906, 2013, p. 21.

Santarosa, L.M.C.; Conforto, D. e Basso L. D. O. "Eduquito: ferramentas de autoria e de colaboração acessíveis na perspectiva da web 2.0". In: Revista Brasileira de Educação Especial, v. 18, n. 3, 2012. ISSN 1413-6538.

Sathiyanarayanan, M. e Rajan, S. "Myo Armband for Physiotherapy Healthcare: A Case Study Using Gesture Recognition Application”. In: International Conference on COMmunication Systems \& NETworks. 8. Bangalore: Índia, 2016. 